\title{
Conservative Finite Difference Formulations, Variable Coefficients, Energy Estimates and Artificial Dissipation
}

Jan Nordström

The self-archived postprint version of this journal article is available at Linköping University Institutional Repository (DiVA):

http://urn.kb.se/resolve?urn=urn:nbn:se:liu:diva-68582

N.B.: When citing this work, cite the original publication.

The original publication is available at www.springerlink.com:

Nordström, J., (2006), Conservative Finite Difference Formulations, Variable Coefficients, Energy Estimates and Artificial Dissipation, Journal of Scientific Computing, 29, 375-404. https://doi.org/10.1007/s10915-005-9013-4

Original publication available at:

https://doi.org/10.1007/s10915-005-9013-4

Copyright: Springer Verlag (Germany)

http://www.springerlink.com/?MUD=MP 


\title{
Conservative Finite Difference Formulations, Variable Coefficients, Energy Estimates and Artificial Dissipation
}

\author{
Jan Nordström ${ }^{1}$
}

\begin{abstract}
Artificial dissipation terms for finite difference approximations of linear hyperbolic problems with variable coefficients are determined such that an energy estimate and strict stability is obtained. Both conservative and non-conservative approximations are considered. The dissipation terms are computed such that there is no loss of accuracy.
\end{abstract}

KEY WORDS: Artificial dissipation; finite differences; stability; variable coefficients; energy estimate.

\section{INTRODUCTION}

Most difference methods for solving nonlinear hyperbolic problems are on conservative form. Conservation is required for a correct shock speed in a nonlinear problem, see [7]. For variable coefficient problems both conservative and non-conservative formulations are used. Examples of important variable coefficient problems in applications include aeroacoustic (the linearized Euler equations), electro-magnetics (variable permittivity and permeability in the Maxwell's equations) and problems where curvilinear meshes with varying metric coefficients are used.

Normally, the artificial dissipation is constructed to absorb the energy of unresolved modes in the problem. It can also be added to enable the calculation of problems involving shocks (see [9] for a discussion on artificial dissipation operators). In this paper we aim for a particular kind of

\footnotetext{
${ }^{1}$ The Swedish Defence Research Agency, Division of Systems Technology, Computational Physics Department; and the Department of Scientific Computing, Information Technology, Uppsala University, Uppsala, Sweden. E-mail: Jan.Nordstrom@foi.se.
} 
artificial dissipation that makes it possible to obtain an energy estimate despite a basic conservative (or non-conservative) difference approximation. Other authors have studied this problem as well, see for example [5] and references therein.

An energy estimate is necessary (but not sufficient) for obtaining the correct long time behavior and strict stability of the scheme. The artificial dissipation derived in this paper is constructed by expressing the conservative formulation as a skew-symmetric formulation with an artificial dissipation term added to it. The size and form of the artificial dissipation term depend on the specific problem to be solved, the size of the mesh and the order of accuracy of the difference operators we use. By observing the behavior of the new conservative scheme and comparing it to the skewsymmetric one, we arrive at the conclusion that the additional requirement for strict stability is a certain amount of smoothness in the computed solution.

The artificial dissipation discussed in this paper is virtually independent of specific boundary treatments and therefore those problems are to a large extent ignored in this paper.

\section{SPATIAL APPROXIMATIONS AND ENERGY ESTIMATES}

Consider the problem,

$$
u_{t}+a(b u)_{x}=0, \quad 0 \leqslant x \leqslant 1, \quad t \geqslant 0,
$$

where $a=a(x)$ and $b=b(x)$. Note that $a=1$ yields a fully conservative formulation. Throughout this paper we assume that the variable coefficients $a$ and $b$ and the solution $u$ are smooth.

Problems on the form (1) typically appear when constant coefficient problems are approximated on a domain using a curvilinear mesh. As was mentioned above, other typical examples on the form (1) include the linearized Euler and Maxwell's equations.

To obtain an energy estimate we can multiply with the solution and integrate over the domain. However, it is instructive to first split the spatial operator into a symmetric and anti-symmetric part. The ansatz

$$
a(b u)_{x}=\alpha(a b u)_{x}+\beta a b u_{x}+\gamma a_{x} b u+\theta a b_{x} u
$$

and accuracy requirements yields $\beta=1-\alpha, \gamma=-\alpha, \theta=1-\alpha$. The first two terms $\left(\alpha(a b u)_{x}+\beta a b u_{x}\right)$ in the spatial differential operator constitute the anti-symmetric part and last two $\left(\gamma a_{x} b u+\theta a b_{x} u\right)$ form the symmetric portion. 
The energy-method applied to (1) with the splitting (2) now yields

$$
\frac{d}{d t}\|u\|_{\ell_{2}}^{2}+\left.a b u^{2}\right|_{0} ^{1}=\int_{0}^{1}\left(a_{x} b-a b_{x}\right) u^{2} d x,
$$

with the choice $\alpha=1 / 2$. In (3) we have introduced the norm $\|u\|_{\ell_{2}}^{2}=$ $\int_{0}^{1} u^{2} d x$.

Remark. Note that (2) is an identity and that the splitting merely exemplifies the fact that only the symmetric part of the spatial differential operator contribute to the estimate.

\subsection{Galerkin Approximations}

For numerical approximations based on the Galerkin approach, the splitting technique used above is not necessary for obtaining an energy estimate. Let

$$
v=L^{T} \alpha=\sum_{i=0}^{i=N} \alpha_{i}(t) L_{i}(x),
$$

approximate $u$. By inserting (4) into (1), multiplying with $L$ and integrating over the domain we get,

$$
P \alpha_{t}+Q \alpha=0
$$

where

$$
P=\int_{0}^{1} L L^{T} d x, \quad Q=Q(a, b)=\int_{0}^{1} L a\left(b L^{T}\right)_{x} d x .
$$

Note that $P$ is a symmetric positive definite matrix and that the dependence of the variable coefficients $a, b$ are built into the operator $Q$.

By using the scheme (5),(6) we will get a perfect energy estimate since

$$
Q=\frac{1}{2}\left(\left.L a b L^{T}\right|_{0} ^{1}+\int_{0}^{1} L a b L_{x}^{T}-L_{x} a b L^{T} d x+\int_{0}^{1} L a b_{x} L^{T}-L a_{x} b L^{T} d x\right) .
$$

Upon multiplying (5) with $\alpha^{T}$ and using (6), (7) we obtain

$$
\frac{d}{d t}\left(\alpha^{T} P \alpha\right)+\alpha^{T}\left(\left.L a b L^{T}\right|_{0} ^{1}\right) \alpha=\alpha^{T}\left(\int_{0}^{1} L a_{x} b L^{T}-L a b_{x} L^{T} d x\right) \alpha,
$$

which exactly correspond to (3) by the use of (4). Note that $Q$ is almost skew-symmetric if $a, b$ are constants. 


\subsection{Finite Difference and Collocation Methods}

Next we turn to finite difference approximations (or spectral/polynomial approximations of collocation type) where the spatial difference operator $D$ is of the summation-by-parts (SBP) type $[2,4,8,10]$. An SBP operator $D$ can be written as a product between two matrices, $P^{-1} Q$ that satisfy the following properties:

1. $P$ is symmetric and positive definite, and $\Delta x p I \leqslant P \leqslant \Delta x q I$, where $p>0$ and $q>0$, are independent of the number of node points, $N+1$.

2. $Q$ is nearly skew symmetric, i.e. $Q+Q^{T}=\operatorname{diag}(-1 \quad 0 \ldots 0 \quad 1)=\mathcal{B}$.

In the remainder of the paper we will only consider diagonal norms $P$ that commutes with varying diagonal matrices.

Remark. If $L_{i}$ in (4) denotes the $i$ th Lagrange polynomial and $a=$ $b=1$, then $P, Q$ in (6) combine to an SBP operator $D=P^{-1} Q$, see [2].

Let $\mathbf{u}$ be the discrete approximation of $u$. The matrices $A, B, A_{x}, B_{x}$ are diagonal with $a\left(x_{i}\right), b\left(x_{i}\right), a_{x}\left(x_{i}\right), b_{x}\left(x_{i}\right)$ as diagonal entries respectively and they commute with $P$. The scalar product is $(\mathbf{u}, \mathbf{v})=\mathbf{u}^{T} P \mathbf{v}$ and the corresponding norm $\|\mathbf{u}\|_{P}^{2}=(\mathbf{u}, \mathbf{u})$.

A direct application of the finite difference (or spectral collocation) technique to (1) yields

$$
\mathbf{u}_{t}+A P^{-1} Q(B \mathbf{u})=0 .
$$

By multiplying (9) with $\mathbf{u}^{T} P$ we obtain

$$
\frac{d}{d t}\|\mathbf{u}\|_{P}^{2}+\mathbf{u}^{T} \mathcal{B} A B \mathbf{u}=(D(A \mathbf{u}), B \mathbf{u})-(A \mathbf{u}, D(B \mathbf{u})) .
$$

The right hand side of (10) does not have the form required to obtain an energy estimate.

In the approximation

$$
\mathbf{u}_{t}+\frac{1}{2} P^{-1} Q(A B \mathbf{u})+\frac{1}{2} A B P^{-1} Q \mathbf{u}-\frac{1}{2}\left(A_{x} B-A B_{x}\right) \mathbf{u}=0,
$$

the same splitting as in (2) is used. The energy estimate becomes

$$
\frac{d}{d t}\|\mathbf{u}\|_{P}^{2}+\mathbf{u}^{T} \mathcal{B} A B \mathbf{u}=\left(\mathbf{u},\left(A_{x} B-A B_{x}\right) \mathbf{u}\right),
$$

which exactly mimics the continuous estimate (3). 
Remark. In contrast to the Galerkin approach, the splitting technique is required in order to obtain an energy estimate for the finite difference method. Note that the compact formulation of $Q(a, b)$ in (6) for the Galerkin approach correspond to

$$
\frac{1}{2} Q(A B \mathbf{u})+\frac{1}{2} A B Q \mathbf{u}-\frac{1}{2} P\left(A_{x} B-A B_{x}\right) \mathbf{u}
$$

in the finite difference (spectral collocation) case.

\subsection{Stability}

Consider the following initial-boundary problem.

$$
\begin{aligned}
u_{t}+H\left(x, t, \frac{\partial}{\partial x_{i}}\right) u & =F(x, t) & & x \in \Omega, \quad t \geqslant 0 \\
u & =f(x) & & x \in \Omega, \quad t=0 \\
L u & =g(t) & & x \in \Gamma, \quad t \geqslant 0
\end{aligned}
$$

where $i=1,2,3$ and $H, L$ are the spatial and boundary operator respectively. $F, f, g$ are the forcing function, initial data and boundary data respectively.

Definition 1. (13) is said to be strongly well posed if an unique solution exists and the estimate

$$
\|u\|_{\Omega}^{2}+\int_{0}^{t}\|u\|_{\Gamma}^{2} d \tau \leqslant K_{c} e^{\eta_{c} t}\left(\|f\|_{\Omega}^{2}+\int_{0}^{t}\left(\|F\|_{\Omega}^{2}+\|g\|_{\Gamma}^{2}\right) d \tau\right)
$$

holds. $K_{c}$ and $\eta_{c}$ do not depend on $F, f$ or $g .\|\cdot\|_{\Gamma}$ and $\|\cdot\|_{\Omega}$ are suitable continuous norms.

The corresponding semi-discrete problem is

$$
\begin{aligned}
\mathbf{u}_{t}+\mathbf{H}\left(x, t, D_{i}\right) \mathbf{u} & =\mathbf{F}(t) & & x \in \Omega, \quad t \geqslant 0 \\
\mathbf{u} & =\mathbf{f} & & x \in \Omega, \quad t=0 \\
\mathbf{L u} & =\mathbf{g}(t) & & x \in \Gamma, \quad t \geqslant 0
\end{aligned}
$$

where $D_{i}$ is the difference operator that approximates $\frac{\partial}{\partial x_{i}}, i=1,2,3$.

Definition 2. (15) is said to be strongly stable if, for a sufficiently small $\Delta x$, there is an unique solution that satisfies

$$
\|\mathbf{u}\|_{\Omega}^{h 2}+\int_{0}^{t}\|\mathbf{u}\|_{\Gamma}^{h 2} d \tau \leqslant K_{d} e^{\eta_{d} t}\left(\|\mathbf{u}\|_{\Omega}^{h 2}+\int_{0}^{t}\left(\|\mathbf{F}\|_{\Omega}^{h 2}+\|\mathbf{g}\|_{\Gamma}^{h 2}\right) d \tau\right) .
$$


$K_{d}$ and $\eta_{d}$ do not depend on $\mathbf{F}, \mathbf{f}$ or $\mathbf{g} .\|\cdot\|_{\Gamma}^{h}$ and $\|\cdot\|_{\Omega}^{h}$ are suitable discrete norms.

Definition 3. We call (15) strictly stable if the growth rates in (14) and (16) satisfy

$$
\eta_{d} \leqslant \eta_{c}+O(\Delta x)
$$

Remark. For a constant coefficient problem where $H$ does not depend on $x$ we expect that $\eta_{d} \leqslant \eta_{c}$, for more details see [6]. A strictly stable scheme (the growth/decay rate for the discrete problem is bounded by the growth/decay rate of the continuous problem) is very important for long time calculations. This property guarantees that high frequency errors with low energy content do not grow and destroy the accuracy of a long time calculation for realistic meshes.

\section{THE LINEAR PROBLEM}

In the rest of this paper we will consider a special model problem constructed to 1) give an energy estimate independent of the boundary conditions and 2) enable an exact calculation of the spectrum of the continuous problem. In the remainder of this paper we will deal with conservative approximations only, non-conservative formulations, see (3), adds no extra difficulties.

Consider the linear system of equations on conservation form,

$$
\begin{aligned}
& u_{t}+(a(x) u)_{x}=0 \\
& v_{t}+(b(x) v)_{x}=0
\end{aligned}
$$

where $a(x)>0, b(x)<0, x \in\left[\begin{array}{ll}0 & 1\end{array}\right]$ and $t>0$. The boundary conditions are determined by

$$
u(0, t)=\alpha v(0, t), \quad v(1, t)=\beta u(1, t)
$$

and the initial conditions are $u(x, 0)=f(x), v(x, 0)=g(x)$.

Multiplication of (18) with $u, v$ and integration over the domain leads to

$$
\frac{d}{d t}\left(\|u\|_{\ell_{2}}^{2}+\|v\|_{\ell_{2}}^{2}\right)=-\int_{0}^{1}\left(a_{x} u^{2}+b_{x} v^{2}\right) d x
$$

where we have introduced $\alpha=\sqrt{-b(0) / a(0)}$ and $\beta=\sqrt{-a(1) / b(1)}$ in order to eliminate the boundary terms. 


\subsection{A Conservative Approximation}

A semi-discrete representation of (18) and (19) is

$$
\begin{aligned}
\mathbf{u}_{t}+D(A \mathbf{u}) & =0 \\
\mathbf{v}_{t}+D(B \mathbf{v}) & =0 \\
u_{0}(t) & =\alpha v_{0}(t) \\
v_{N}(t) & =\beta u_{N}(t),
\end{aligned}
$$

augmented with initial conditions. A and $\mathrm{B}$ are diagonal matrices with the values of $a$ and $b$ injected on the diagonal.

The system (21) is obviously on conservation form since if we multiply with a smooth function $\phi$ we obtain

$$
(\phi, D(A u))=\phi^{T} P D(A u)=\phi^{T} Q A u=\phi^{T} \mathcal{B} A u-((D \phi), A u)
$$

by using the summation by parts properties mentioned in the previous section. Let $\mathbf{w}=(\mathbf{u}, \mathbf{v})^{T}$,

$$
\mathcal{P}=\left(\begin{array}{ll}
P & 0 \\
0 & P
\end{array}\right), \quad \mathcal{Q}=\left(\begin{array}{ll}
Q & 0 \\
0 & Q
\end{array}\right), \quad \mathcal{F}=\left(\begin{array}{cc}
A & 0 \\
0 & B
\end{array}\right),
$$

and $\mathcal{B}=\mathcal{Q}+\mathcal{Q}^{T}$ (a slight abuse of notation since $\mathcal{B}$ was used also for the scalar case). Using the Simultaneous approximation term (SAT) method [3], which makes the boundary conditions part of the difference equation through a "penalty"-term, and the definitions in (22) we can write (21) as

$$
\mathbf{w}_{t}+\mathcal{D} \mathcal{F}_{\mathbf{w}}=\mathcal{P}^{-1} S \mathbf{w},
$$

where $\mathcal{D}=\mathcal{P}^{-1} \mathcal{Q}$ and $S$ is a $(2 N+2) *(2 N+2)$ matrix with nonzero elements at position 1 and $N+2$ in the first row and $N+1$ and $2 N+2$ in the last row.

$$
S=\left(\begin{array}{cccccc}
\sigma_{L} & 0 & \ldots & -\sigma_{L} \alpha & 0 & \ldots \\
0 & 0 & & & & \\
\vdots & & \ddots & & & \\
& & & & & \vdots \\
& & & & 0 & 0 \\
& & -\sigma_{R} \beta & 0 & \ldots & \sigma_{R}
\end{array}\right) .
$$

Multiplying equation (23) with $\mathbf{w}^{T} \mathcal{P}$ from the left and adding the transposed resulting equation results in

$$
\mathbf{w}^{T} \mathcal{P} \mathbf{w}_{t}+\mathbf{w}_{t}^{T} \mathcal{P} \mathbf{w}=\mathbf{w}^{T}\left(S+S^{T}\right) \mathbf{w}-\mathbf{w}^{T}\left(\mathcal{Q F}+(\mathcal{Q F})^{T}\right) \mathbf{w} .
$$


By using $\mathcal{Q}+\mathcal{Q}^{T}=\mathcal{B}$ we get

$$
\frac{d}{d t}\|\mathbf{w}\|_{\mathcal{P}}^{2}=B T_{1}+\left(\mathcal{D} \mathbf{w}, \mathcal{F}_{\mathbf{w}}\right)_{\mathcal{P}}-(\mathcal{D} \mathcal{F} \mathbf{w}, \mathbf{w})_{\mathcal{P}}
$$

where $B T_{1}=\mathbf{w}^{T}\left(S+S^{T}-\mathcal{B F}\right) \mathbf{w}$ and $(\mathbf{u}, \mathbf{v})=\mathbf{u}^{T} P \mathbf{v}$. Let

$$
G R 1=(\mathcal{D} \mathbf{w}, \mathcal{F} \mathbf{w})_{\mathcal{P}}-(\mathcal{D} \mathcal{F} \mathbf{w}, \mathbf{w})_{\mathcal{P}}
$$

To obtain strict stability, a first requirement is that $B T_{1}$ has to be less than or equal to zero. The choice

$$
\sigma_{L}=-a_{0}, \quad \sigma_{R}=-b_{N},
$$

leads to non-positive eigenvalues of $\left(S+S^{T}-\mathcal{B F}\right)$.

\subsection{A Skew-Symmetric Approximation}

Another approximation of (18) is obtained by using the splitting technique described in section 2 . We get

$$
\begin{aligned}
& \mathbf{u}_{t}+\frac{1}{2}(D A \mathbf{u}+A D \mathbf{u})+\frac{1}{2} A_{x} \mathbf{u}=0 \\
& \mathbf{v}_{t}+\frac{1}{2}(D B \mathbf{v}+B D \mathbf{v})+\frac{1}{2} B_{x} \mathbf{v}=0,
\end{aligned}
$$

which is equivalent to

$$
\mathbf{w}_{t}+\frac{1}{2}\left(\mathcal{F}_{x} \mathbf{w}+\mathcal{D} \mathcal{F} \mathbf{w}+\mathcal{F} \mathcal{D} \mathbf{w}\right)=0 .
$$

In (26), $\mathcal{F}_{x}=\operatorname{diag}\left(A_{x}, B_{x}\right)$ and $A_{x}, B_{x}$ have the values of $a_{x}, b_{x}$ injected on the diagonal. Equation (26) augmented with the SAT term becomes

$$
\mathbf{w}_{t}+\frac{1}{2} \mathcal{F}_{x} \mathbf{w}+\frac{1}{2}(\mathcal{D} \mathcal{F} \mathbf{w}+\mathcal{F} \mathcal{D} \mathbf{w})=\mathcal{P}^{-1} S \mathbf{w},
$$

where the matrix $S$ is the same as in Sec. 3.1. By multiplying (27) with $\mathbf{w}^{T} \mathcal{P}$ from the left and then adding the corresponding transposed equation, we get

$$
\begin{aligned}
\mathbf{w}^{T} \mathcal{P} \mathbf{w}_{t}+\mathbf{w}_{t}^{T} \mathcal{P} \mathbf{w}=\mathbf{w}^{T}\left(S+S^{T}\right) \mathbf{w}-\frac{1}{2} \mathbf{w}^{T}\left(\mathcal{P} \mathcal{F}_{x}+\left(\mathcal{P} \mathcal{F}_{x}\right)^{T}\right) \mathbf{w} \\
-\frac{1}{2} \mathbf{w}^{T}\left(\mathcal{Q} \mathcal{F}+\mathcal{F}^{T} \mathcal{Q}^{T}+\mathcal{P} \mathcal{F} \mathcal{D}+\mathcal{P} \mathcal{F} \mathcal{D}^{T}\right) \mathbf{w} .
\end{aligned}
$$

Now since $\mathcal{P}$ and $\mathcal{F}$ commute we get $\mathcal{P F D}=\mathcal{P F P}{ }^{-1} \mathcal{Q}=\mathcal{F Q}$ which leads to

$$
\frac{d}{d t}\|\mathbf{w}\|_{\mathcal{P}}^{2}=B T_{2}-\left(\mathcal{F}_{x} \mathbf{w}, \mathbf{w}\right)_{\mathcal{P}} .
$$


The boundary term $B T_{2}=\mathbf{w}^{T}\left(S+S^{T}-\mathcal{B F}\right) \mathbf{w}$ equals $B T_{1}$ in section 3.1 and is therefore negative semi definite if $(25)$ is used. Let

$$
G R 2=-\left(\mathcal{F}_{x} \mathbf{w}, \mathbf{w}\right)_{\mathcal{P}}
$$

\subsection{The Energy Estimate}

To obtain an energy estimate where the growth rate corresponds to the continuous case, $G R 1$ in (24) for the conservative formulation and $G R 2$ in (29) for skew-symmetric formulation must correspond to the righthand side in (20). Otherwise, strict stability will not be obtained.

Using a second order SBP operator, $a(x)=1+\epsilon x$ and $b(x)=-1+\epsilon x$ imply that $G R 2=-\left(\mathcal{F}_{x} \mathbf{w}, \mathbf{w}\right)_{\mathcal{P}}=-\epsilon\|\mathbf{w}\|_{\mathcal{P}}^{2}$. This means that we get

$$
\frac{d}{d t}\|\mathbf{w}\|_{\mathcal{P}}^{2}=-\epsilon\|\mathbf{w}\|_{\mathcal{P}}^{2}
$$

which mimics (20) perfectly and therefore a correct discrete spectrum is obtained, see Figs. 1 and 2.

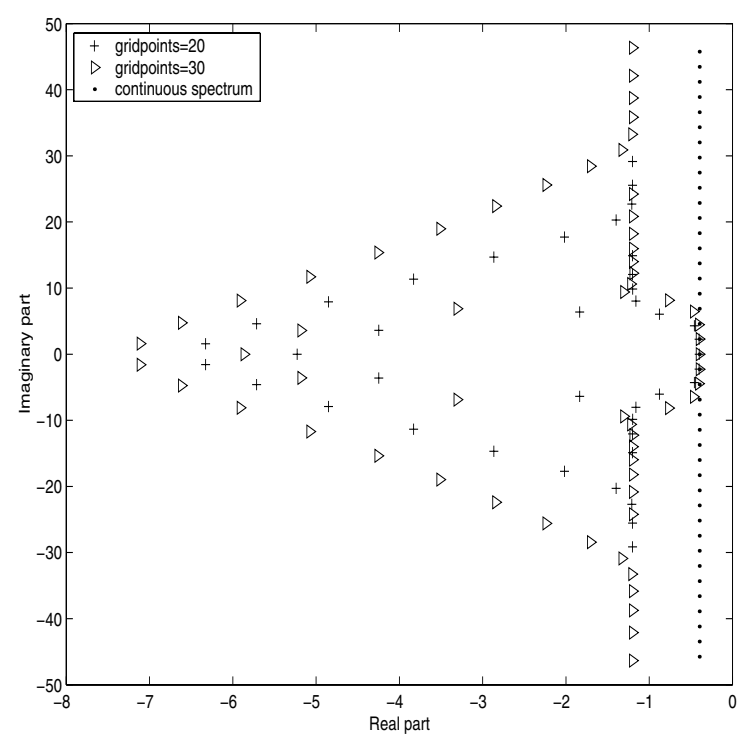

Fig. 1. Second order skew-symmetric case, $a=1+0.8 x, b=-1+0.8 x$. 


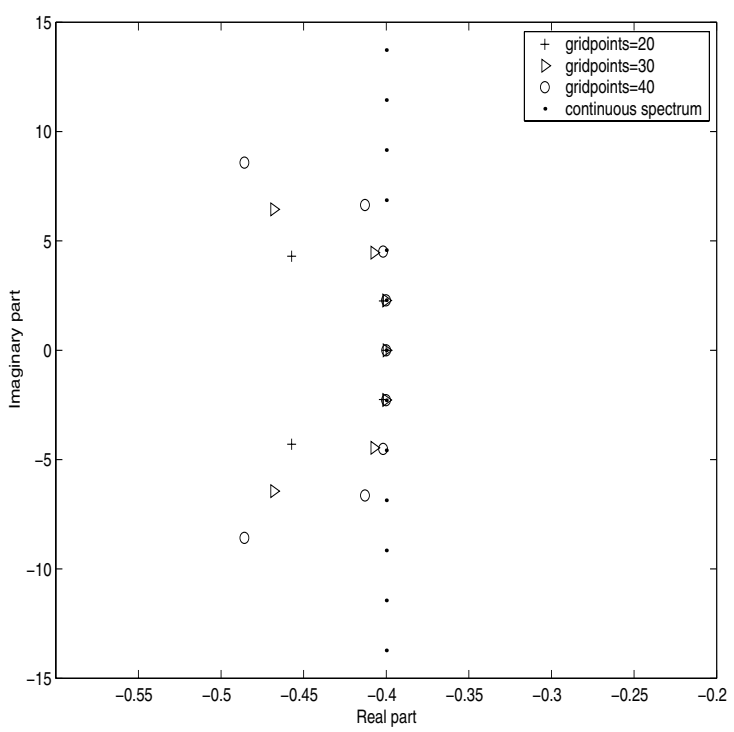

Fig. 2. Close-up, second order skew-symmetric case, $a=1+0.8 x, b=-1+0.8 x$.

In the conservative case we get

$$
G R 1=-\epsilon\|\mathbf{w}\|_{\mathcal{P}}^{2}+E, \quad E=\frac{\epsilon \Delta x}{2} \sum_{i=1}^{N}\left(\left(u_{i-1}-u_{i}\right)^{2}+\left(v_{i-1}-v_{i}\right)^{2}\right) .
$$

The deviation $E>0$ does not necessarily vanish with decreasing $\Delta x$ nor can it be estimated. As an example, assume that the solutions have a smooth (superscript s) and a high frequency oscillatory part (with amplitudes $\alpha, \beta)$, then $u_{i}=u_{i}^{s}+\alpha(-1)^{i}, v_{i}=v_{i}^{s}+\beta(-1)^{i}$. This leads to $E=$ $2 \epsilon\left(\alpha^{2}+\beta^{2}\right)$ for large $N$. If an energy estimate existed, or we for some other reasons knew that the scheme was stable, then $\alpha, \beta$ and also $E$ would go to zero. In this case we have no such knowledge and consequently, the spectrum might not be correct, see Figs. 3 and 4.

Note that if $\mathbf{u}$ and $\mathbf{v}$ in (29) can be interpolated to smooth functions, then

$$
G R 2 \approx-\int_{0}^{1}\left(a_{x} u^{2}+b_{x} v^{2}\right) d x,
$$

which indicates that the energy rate (28) for the skew-symmetric method correspond to (20). 


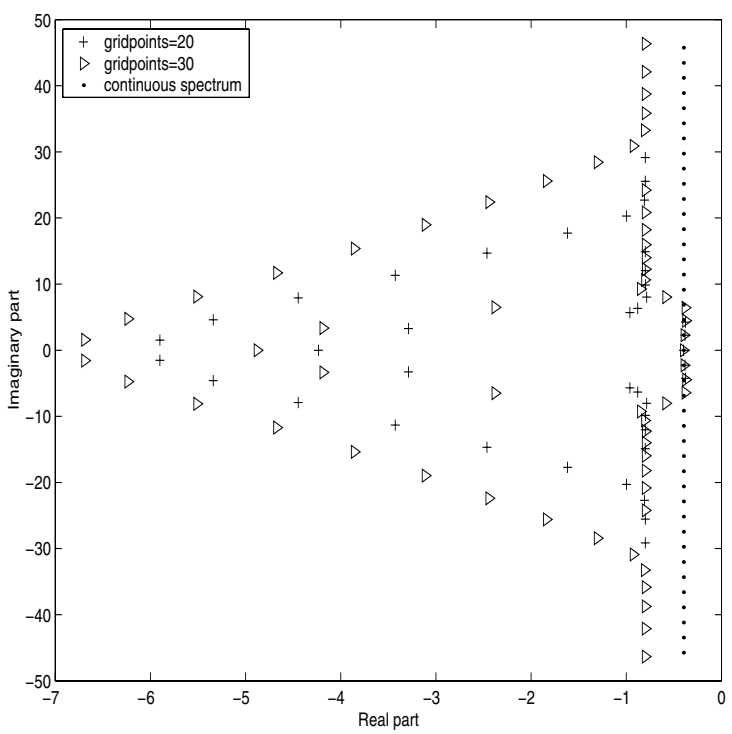

Fig. 3. Second order conservative case, $a=1+0.8 x, b=-1+0.8 x$.

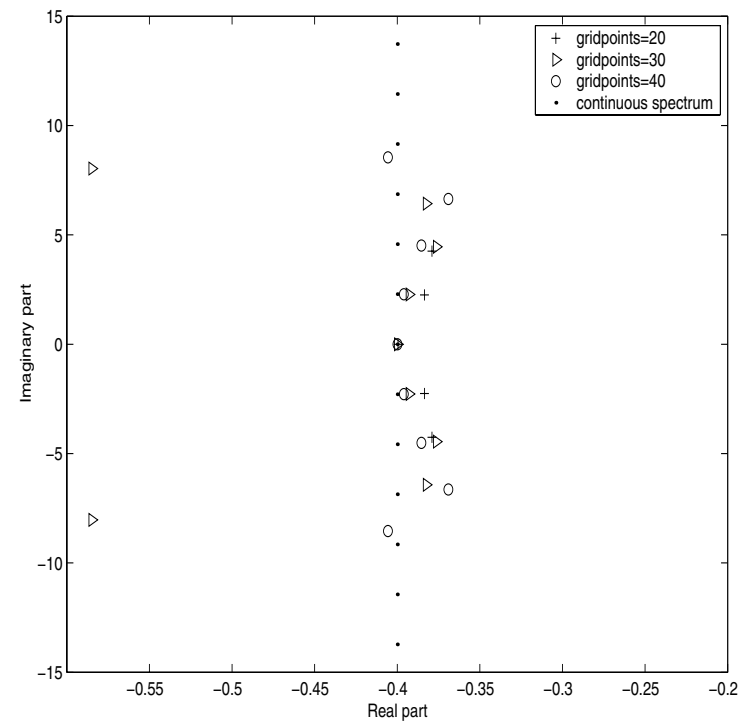

Fig. 4. Close-up, second order conservative case, $a=1+0.8 x, b=-1+0.8 x$. 


\subsection{Continuous and Discrete Spectrum}

By Laplace transforming (18) we get

$$
\begin{aligned}
& s \tilde{u}-f(x)+(a \tilde{u})_{x}=0 \\
& s \tilde{v}-g(x)+(b \tilde{v})_{x}=0,
\end{aligned}
$$

where $\tilde{u}(s)=\int_{0}^{\infty} u e^{-s t} d t$. The solutions to (31) with $f(x)=g(x)=0$, are

$$
\tilde{u}=\frac{C_{1}}{|a| \int_{\delta_{1}}^{x} \frac{s}{a} d x}, \quad \tilde{v}=\frac{C_{2}}{|b| \int_{\delta_{2}}^{x} \frac{s}{b} d x} .
$$

$C_{1}$ and $C_{2}$ are constants and $\delta_{1}$ and $\delta_{2}$ are arbitrary real numbers. The solutions $\tilde{u}$ and $\tilde{v}$ in (32) inserted in the boundary conditions (19) leads to

$$
M\left(\begin{array}{l}
C_{1} \\
C_{2}
\end{array}\right)=\left(\begin{array}{cc}
\tilde{u}_{1}(0, s) & -\alpha \tilde{v}_{1}(0, s) \\
\beta \tilde{u}_{1}(1, s) & -\tilde{v}_{1}(1, s)
\end{array}\right)\left(\begin{array}{l}
C_{1} \\
C_{2}
\end{array}\right)=0
$$

where $\tilde{u}_{1}=\tilde{u} / C_{1}$ and $\tilde{v}_{1}=\tilde{v} / C_{2}$. The spectrum is determined by solving $|M|=0$ for the s values. For general $a(x)$ and $b(x)$ the spectrum is given by

$$
s=\frac{\ln (\alpha \beta)+2 n \pi i}{\int_{1}^{0} a(x)^{-1} d x+\int_{0}^{1} b(x)^{-1} d x},
$$

where $i$ is the imaginary unit and $n \in \mathbb{Z}$.

The discrete spectrum is given by computing the eigenvalues of $G$ in $\mathbf{w}_{t}=G \mathbf{w}$ where

$$
G=-\mathcal{D F}+\mathcal{P}^{-1} S, \quad G=-\frac{1}{2} \mathcal{F}_{x}-\frac{1}{2}(\mathcal{D F}+\mathcal{F} \mathcal{D})+\mathcal{P}^{-1} S,
$$

for the conservative and skew-symmetric formulation respectively.

In Figs. 1 and 2 all eigenvalues converge toward the continuous spectrum from the left. This imply strict stability for the skew-symmetric method and linear variation of the wave speeds $a, b$. For the conservative formulation, see Figs. 3 and 4 we have no such convergence and the method is not strictly stable.

The discrete spectrum in Figs. 5, and 6 for a more general variation of the wave speeds do converge to the continuous spectrum $\lambda_{C}^{\mathrm{Re}}=$ $s$ when refining the grid, but $\left(\lambda_{j}^{\mathrm{Re}}\right)_{\max } j=1, \ldots, N+1$ increases when $\Delta x$ decreases. This means that not even the skew-symmetric formulation is strictly stable, see Definition 3, Eq. (17). 


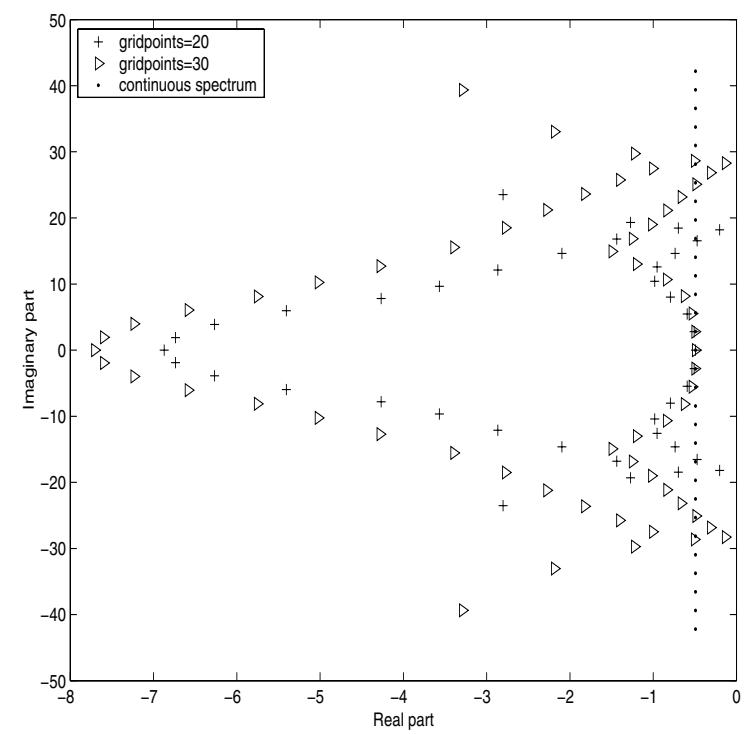

Fig. 5. Second order skew-symmetric case, $a=1+0.8 x^{4}, b=-1+0.8 x^{4}$.

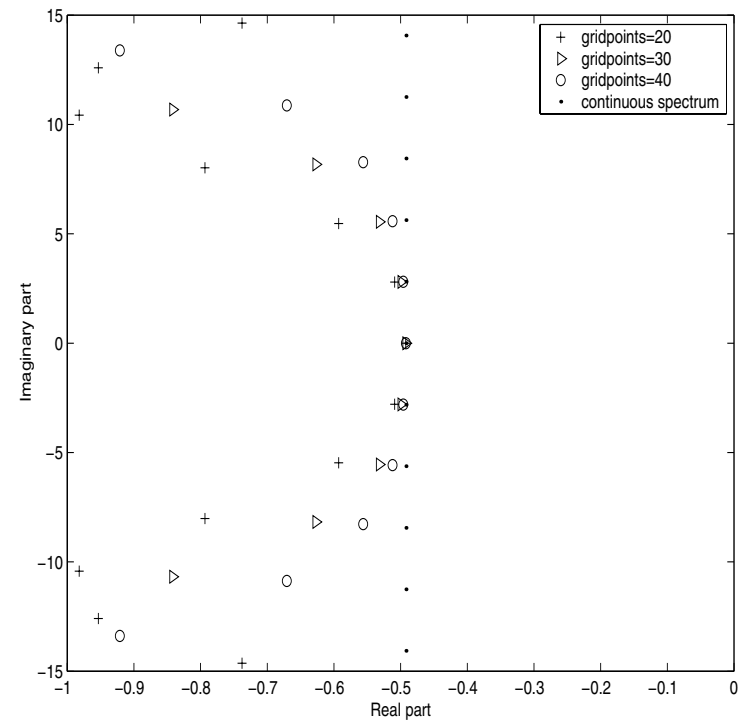

Fig. 6. Close-up, second order skew-symmetric case, $a=1+0.8 x^{4}, b=-1+0.8 x^{4}$. 


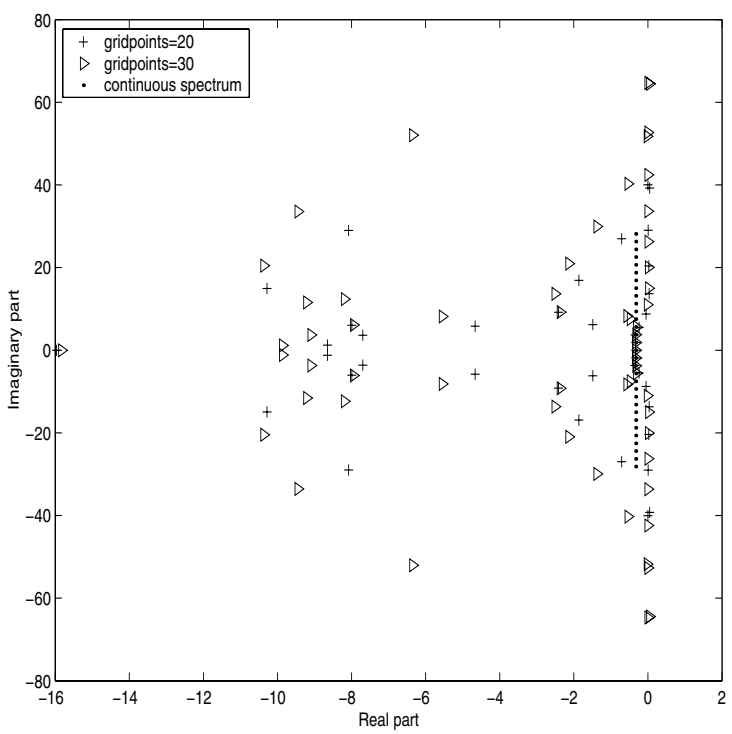

Fig. 7. Fourth order skew-symmetric case, $a=1+0.8 \sin (7.9 x), b=-1+0.8 \sin (7.9 x)$.

In Fig. 7 the continuous spectrum has real parts with negative eigenvalues while in the discrete spectrum, some eigenvalues have positive real parts. That will result in an explosion of the solution as time increases.

The conclusion we can draw from the spectra shown above is that we cannot always guarantee strict stability, not even with a skew-symmetric formulation. However,

$$
\frac{d}{d t}\|\mathbf{w}\|_{\mathcal{P}}^{2}=-\left(\mathcal{F}_{x} \mathbf{w}, \mathbf{w}\right)_{\mathcal{P}} \leqslant\left|\mathcal{F}_{x}\right|_{\max }\|\mathbf{w}\|_{\mathcal{P}}^{2},
$$

is always valid in the skew-symmetric formulation, i.e we can obtain an energy estimate. For the conservative scheme (23) we cannot produce such an estimate.

Remark. The fact that we could not obtain a strictly stable formulation with the skew-symmetric formulation was a surprise for us at this point in time. The results in [1] where coefficients with constant gradients ( $a_{x}=$ const.) were considered lead us to believe that the spectrum would lie in the correct position for a skew-symmetric formulation and general smooth variable coefficients. However, as can be seen in Figs. 5 and 7, that is clearly not the case. We will come back to this point later. 


\subsection{A Conservative Method with Artificial Dissipation}

Although we now have a numerical method with a bounded energy rate, see section 3.2, it might be preferable to solve the problem (18) using a conservative formulation of the problem as in (21) (that is certainly true in the non linear case, see [7]).

Adding and subtracting $\frac{1}{2}\left(A_{x} \mathbf{u}+A \mathcal{D} \mathbf{u}\right)$ and $\frac{1}{2}\left(B_{x} \mathbf{v}+B \mathcal{D} \mathbf{v}\right)$ respectively from the conservative formulation (21) we get

$$
\begin{aligned}
& \mathbf{u}_{t}+D A \mathbf{u}+\frac{1}{2}\left(A_{x} \mathbf{u}+A D \mathbf{u}\right)-\frac{1}{2}\left(A_{x} \mathbf{u}+A D \mathbf{u}\right)=0 \\
& \mathbf{v}_{t}+D B \mathbf{v}+\frac{1}{2}\left(B_{x} \mathbf{v}+B D \mathbf{v}\right)-\frac{1}{2}\left(B_{x} \mathbf{v}+B D \mathbf{v}\right)=0 .
\end{aligned}
$$

After rearranging the terms in (34) and by using (22) the semi-discrete problem can be written,

$$
\mathbf{w}_{t}+\frac{1}{2}\left(\mathcal{F}_{x} \mathbf{w}+\mathcal{D} \mathcal{F} \mathbf{w}+\mathcal{F} \mathcal{D} \mathbf{w}\right)=R, \quad R=\frac{1}{2}\left(-\mathcal{D} \mathcal{F} \mathbf{w}+\mathcal{F}_{x} \mathbf{w}+\mathcal{F} \mathcal{D} \mathbf{w}\right),
$$

which is exactly the skew-symmetric formulation (26), except for the term on the right hand side. If $R$ is a non dissipative term we need to dominate it by suitable artificial dissipation terms, preferably without affecting the order of accuracy of the approximation. If we accomplish that, we have a conservative formulation of the problem, that unlike (21),(23) leads to an energy estimate.

For the first equation in (34), $R$ has the form

$$
R_{i}=\frac{1}{2}\left(-D A \mathbf{u}+A_{x} \mathbf{u}+A D \mathbf{u}\right)_{i}, \quad i=\text { the } i \text { th node point. }
$$

The size and form of the artificial dissipation term depends on the spatial difference operator, $D$. In this paper we use second, fourth and sixth order accurate central difference operators (see Appendix A for a description of the first derivative operators).

In the second order case, we get,

$$
D F \approx F_{x}+\frac{(\Delta x)^{2}}{6} F_{x x x}+O\left((\Delta x)^{4}\right) .
$$

By using (37) and (36) we obtain

$$
R_{i} \approx-\frac{(\Delta x)^{2}}{4}\left[\left(a_{x} u_{x}\right)_{x}\right]_{x=x_{i}} .
$$

The contribution to the energy estimate of $R_{i}$ can be estimated by interpreting the result in continuous frame and by multiplying (38) with $u$ and integrate in space. That leads to, 


$$
\int u R d x=\frac{(\Delta x)^{2}}{4} \int a_{x} u_{x}^{2} d x .
$$

According to (39) $R_{i}$ is of a dissipative nature if $a_{x}<0$. To make sure that our artificial dissipation (added to the right hand side of Eq. (23)) is dissipative and large enough to balance $R$, we use the dissipation operators developed in [9] and write this term as

$$
\mathrm{DI} \mathbf{u}=-\frac{(\Delta x)^{2}}{4} \widetilde{P}^{-1} D_{1}^{T} C_{1} D_{1} \mathbf{u} .
$$

In (40), $D_{1}$ approximates a first derivative and $C_{1}=\left|A_{x}\right|_{\max } C$ where $C$ is a diagonal matrix that reduces the values of $\left|A_{x}\right|_{\max }$ at the boundaries to maintain the correct approximation order of the scheme. $\widetilde{P}^{-1}=\Delta x P^{-1}$ is included in order to obtain the correct discrete energy estimate, see [9].

Next we consider the fourth order operator. Taylor expansion and formula (36) yields,

$$
R_{i} \approx \frac{(\Delta x)^{4}}{12}\left[\left(a_{x x x} u_{x}\right)_{x}+\left(a_{x} u_{x x}\right)_{x x}\right]_{x=x_{i}} .
$$

The contribution to the energy rate of $R_{i}$ becomes,

$$
\int u R d x=-\frac{(\Delta x)^{4}}{12} \int a_{x x x} u_{x}^{2} d x+\frac{(\Delta x)^{4}}{12} \int a_{x} u_{x x}^{2} d x .
$$

The first integral in (41) is negative for $a_{x x x}>0$ and the second one for $a_{x}<0$. The dissipation operator we will use becomes

$$
\mathrm{DI}=-\frac{(\Delta x)^{4}}{12} \widetilde{P}^{-1}\left(D_{1}^{T} C_{3} D_{1}+D_{2}^{T} C_{1} D_{2}\right) .
$$

$D_{2}$ in (42) approximates a second derivative and $C_{3}=\left|A_{x x x}\right|_{\max } C$. Both $C_{1}$ and $C$ are defined above.

Finally we consider the sixth order case. Again, Taylor expansion and formula (36) yields,

$$
R_{i} \approx-\frac{(\Delta x)^{6}}{40}\left[\left(a_{x x x x x} u_{x}\right)_{x}+2\left(a_{x x x} u_{x x}\right)_{x x}+\left(a_{x} u_{x x x}\right)_{x x x}\right]_{x=x_{i}}
$$

The contribution to the energy estimate related to $R$ is

$$
\int u R d x=\frac{(\Delta x)^{6}}{40}\left(\int a_{x x x x x} u_{x}^{2} d x-2 \int a_{x x x} u_{x x}^{2} d x+\int a_{x} u_{x x x}^{2} d x\right) .
$$


The dissipation operator is now determined to be

$$
\mathrm{DI}=-\frac{(\Delta x)^{6}}{40} \widetilde{P}^{-1}\left(D_{1}^{T} C_{5} D_{1}+2 D_{2}^{T} C_{3} D_{2}+D_{3}^{T} C_{1} D_{3}\right) .
$$

$D_{3}$ in (44) approximates a third derivative and $C_{5}=\left|A_{x x x x x}\right| \max C . C_{1}, C_{3}$ and $C$ are defined above.

We can summarize the development described above by stating that we have derived an approximation of the form

$$
\mathbf{u}_{t}+D A \mathbf{u}=\mathrm{DI} \mathbf{u},
$$

which we refer to as a conservative approximation with added dissipation (DI). Note the symmetric construction of the dissipation operators in (40), (42) and (44).

Remark. The implementation of this procedure in multiple dimensions is straightforward. One performs the procedure described above and adds on artificial dissipation operators of the form given in (41), (43) and (45) in each coordinate direction, see [9] for more details. In Figs. 8 and 9, the dissipative term has obviously balanced R enough, see (36) and compare with Figs. 5 and 6. Not only do the discrete eigenvalues converge to the continuous ones, but also $\left(\lambda_{j}^{R e}\right)_{\max } j=1, \ldots, N+1$, converges as $\Delta x$ goes to zero. All eigenvalues in Fig. 8 and 9 converge from the left.

As can be seen in Figs. 10 and 11 a higher order of accuracy does not mean that all eigenvalues are closer to the continuous ones. But they do converge faster, which is shown in Figs. 12, 13 and 14.

Eigenvalues for problems involving $a(x)$ and $b(x)$ being polynomials up to fifth degree as well as trigonometric functions have been considered. In all these cases $\left(\lambda_{j}^{\mathrm{Re}}\right)_{\max } j=1, \ldots, N+1$, converges as $\Delta x$ goes to zero for the conservative approximation with the new artificial dissipation, although they sometimes converge from the right.

Remark. Recall that as long as the maximum real part of the numerical eigenvalues converge to the maximum real part of the continuous eigenvalues, see Definition 3, Eq. (17), we have strict stability.

Remark. Now we have a chance to understand why the skew-symmetric formulation did not lead to strict stability while the conservative form with the added dissipation did. A correct spectra would be obtained if

$$
\mathbf{u}^{T} P A_{x} \mathbf{u}=\int_{0}^{1} a_{x} u^{2} d x+O\left(\Delta x^{n}\right)
$$




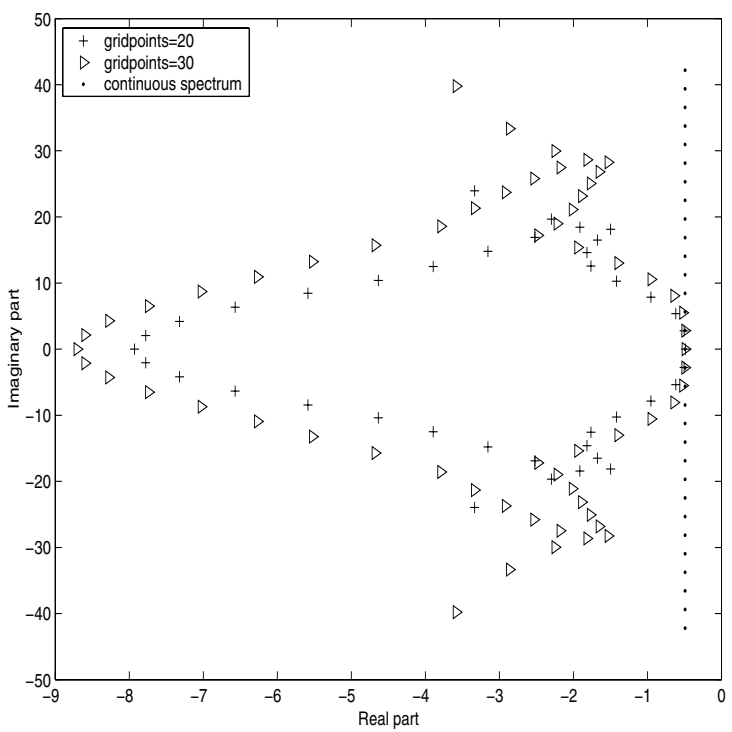

Fig. 8. Conservative method with dissipation term, second order case. $a=1+0.8 x^{4}$, $b=-1+0.8 x^{4}$.

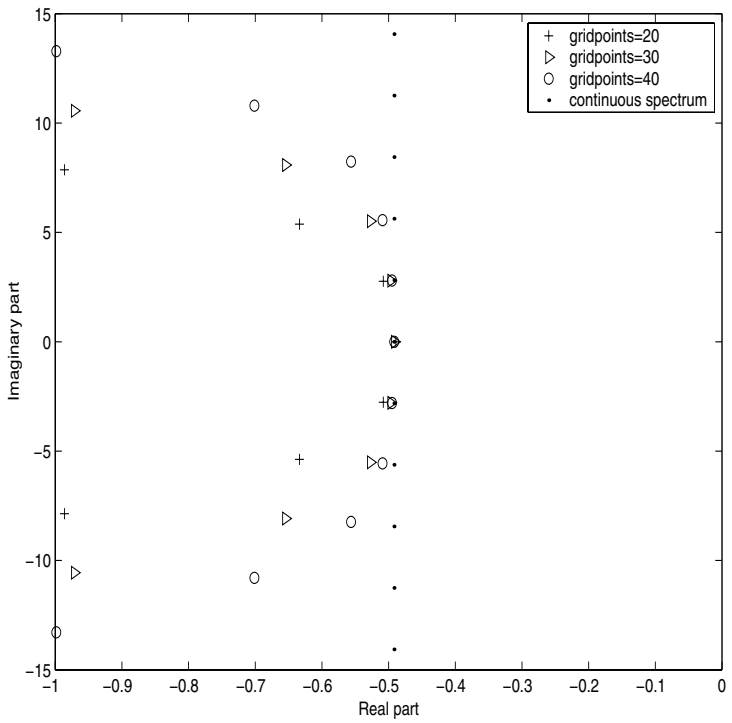

Fig. 9. Close-up, conservative method with dissipation term, second order case. $a=1+$ $0.8 x^{4}, b=-1+0.8 x^{4}$. 


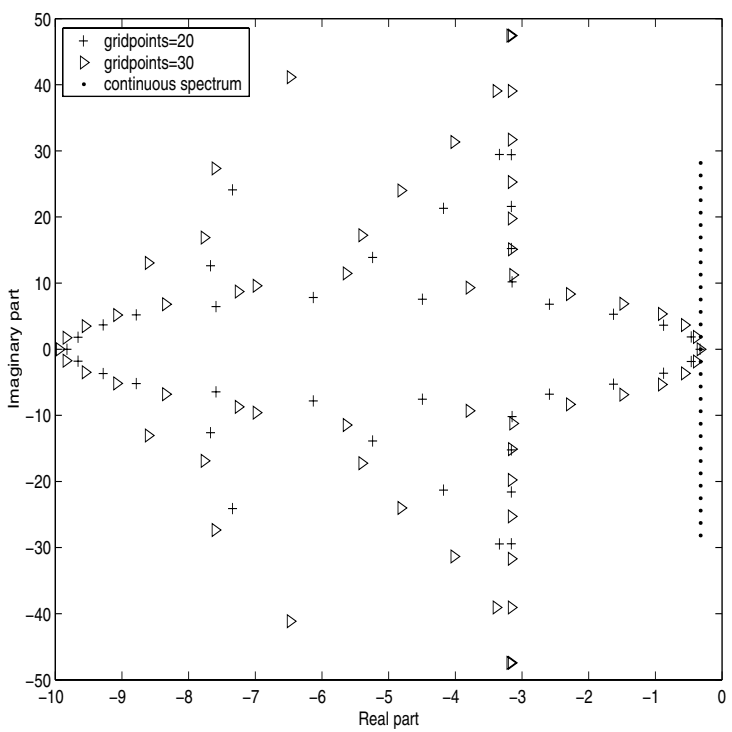

Fig. 10. Conservative method with dissipation term, second order case. $a=1+0.8$ $\sin (7.9 x), b=-1+0.8 \sin (7.9 x)$.

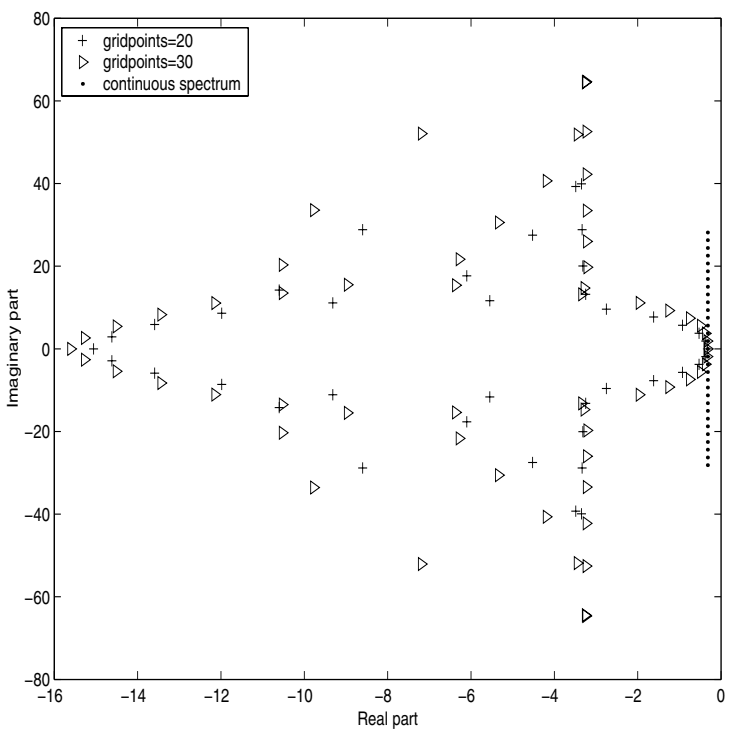

Fig. 11. Conservative method with dissipation term, fourth order case. $a=1+0.8$ $\sin (7.9 x), b=-1+0.8 \sin (7.9 x)$. 


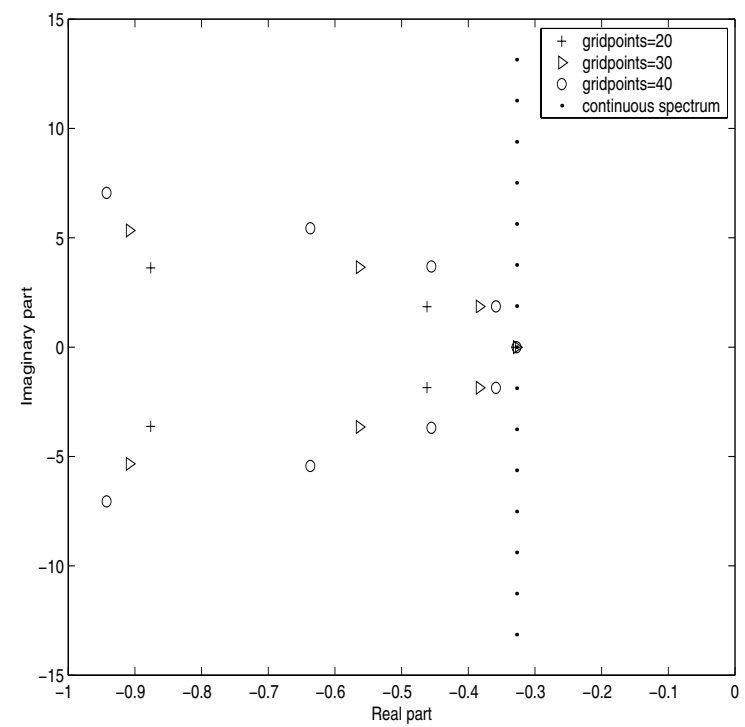

Fig. 12. Close-up, conservative method with dissipation term, second order case. $a=1+0.8$ $\sin (7.9 x), b=-1+0.8 \sin (7.9 x)$.

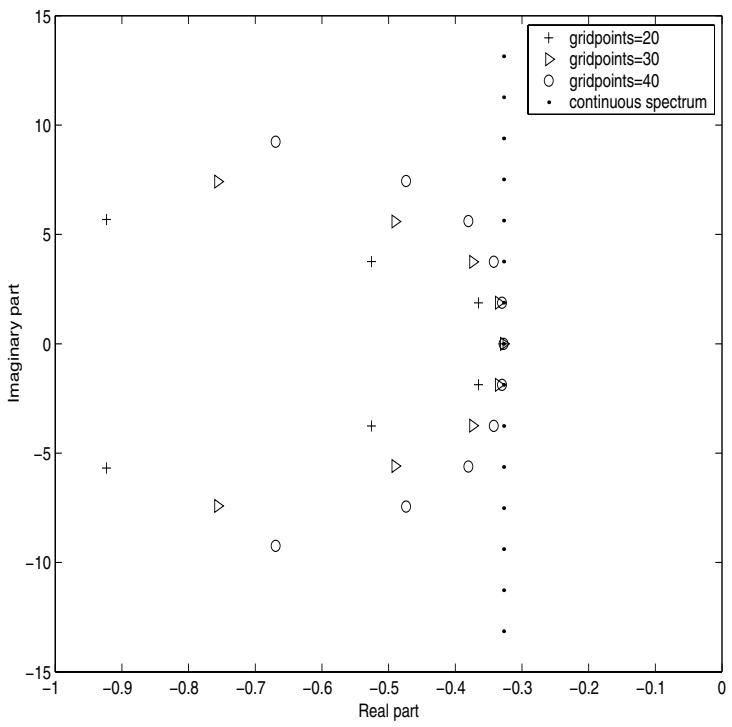

Fig. 13. Close-up, conservative method with dissipation term, fourth order case. $a=1+0.8$ $\sin (7.9 x), b=-1+0.8 \sin (7.9 x)$. 


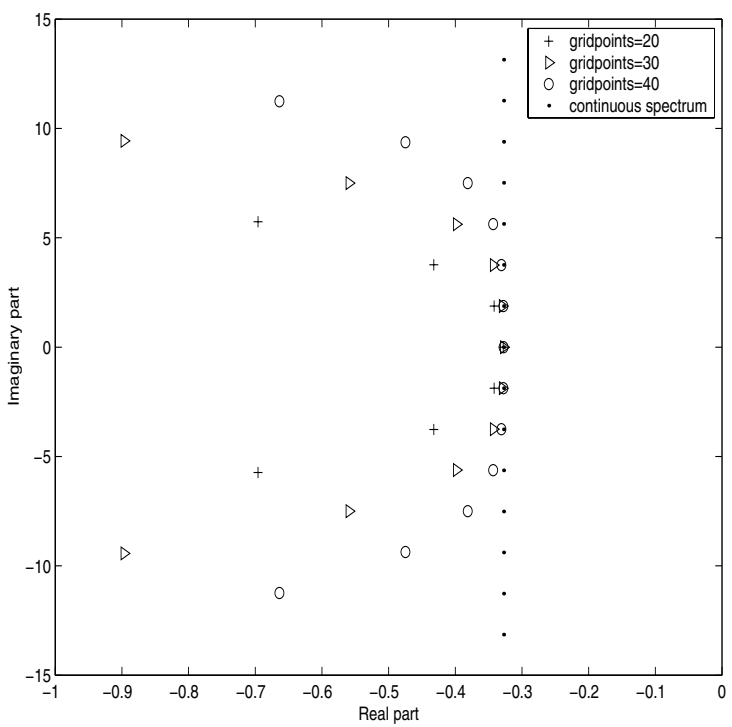

Fig. 14. Close-up, conservative method with dissipation term, sixth order case. $a=1+0.8$ $\sin (7.9 x), b=-1+0.8 \sin (7.9 x)$.

where $n$ is the approximation order of the integration operator $P$. For a smooth function $\mathbf{u}$ we certainly have convergence. However, the solutions in the skew-symmetric cases discussed above were probably not smooth. The conservative form with added dissipation is by construction more dissipative (choice of $\left|A_{x}\right|_{\max }$ ) and hence might lead to smooth solutions and strict stability.

\section{NUMERICAL EXPERIMENTS}

Consider the problem (21). We have chosen to investigate a few cases where we are able to determine the solution analytically, see Appendix B. Second, fourth and sixth order accurate finite difference operators on summation-by-parts (SBP) form (see $[4,8,10]$ ) with diagonal norms are used in the numerical experiments. To integrate in time we use a fourth order accurate Runge-Kutta method.

In Fig. 15 we show results using second, fourth and sixth order accurate approximations an well as the exact result. Note the significant difference in accuracy. Note also that since $a_{x}$ and $b_{x}$, in Fig. 15 alternate between positive and negative numbers, the amplitude of the solution might grow in time, see Eq. (20). 

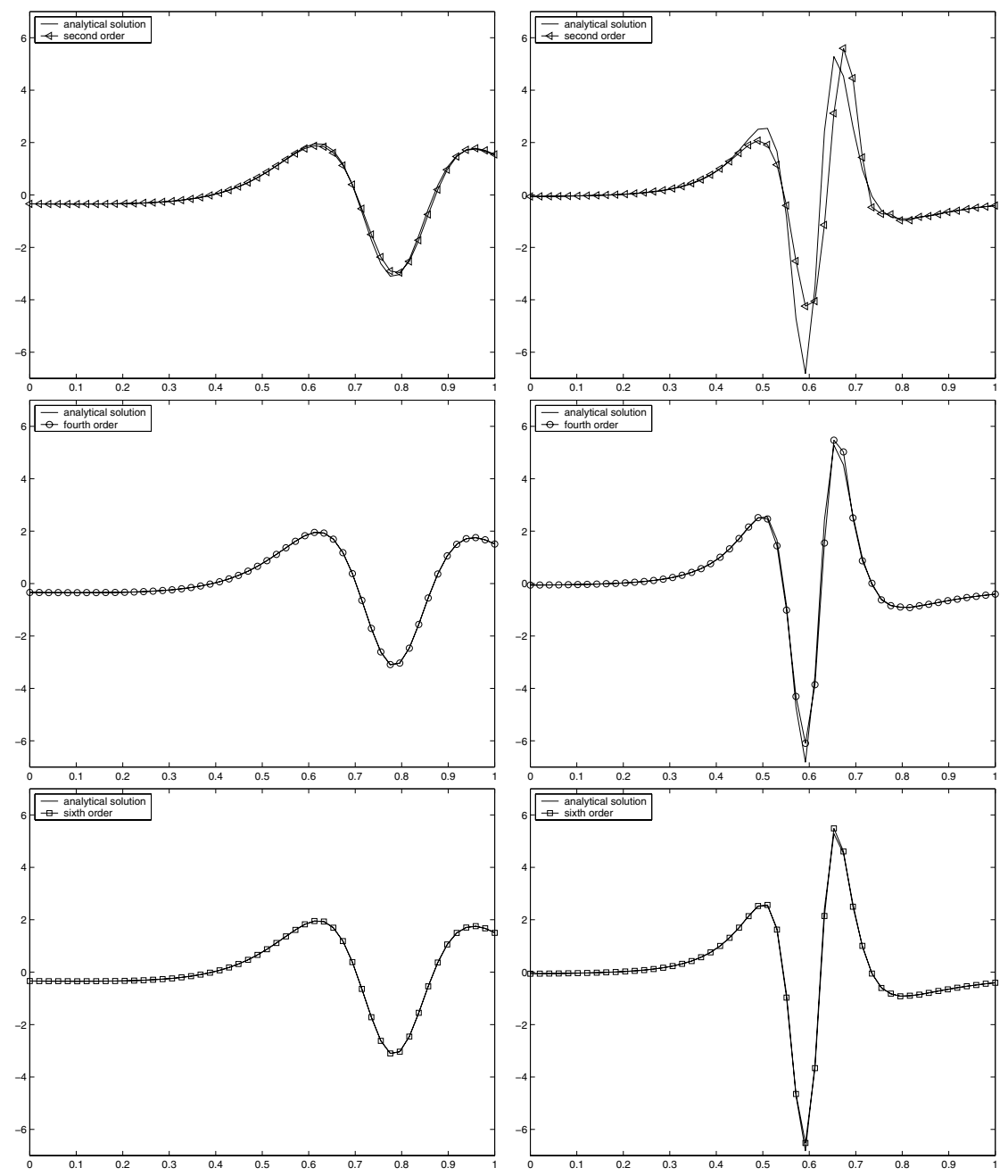

Fig. 15. (a) $v$ at $t=0.5$ and (b) $t=1.1$ for second order case. (c) $v$ at $t=0.5$ and (d) $t=1.1$ for fourth order case. (e) $v$ at $t=0.5$ and (f) $t=1.1$ for sixth order case. $a=1+0.8 \sin (\pi x)$, $b=-1+0.8 \sin (\pi x), f=\sin (2 \pi x), g=-f, N=50$.

Since SBP-operators with diagonal norms are used, the order of accuracy at the boundary is half the one used in the interior of the domain, see [4]. This implies that the total order of accuracy in space becomes 2, 3 and 4 for the second, fourth and sixth order schemes respectively. The order of actuary is not altered by the artificial dissipation, which can be seen in Fig. 16. 


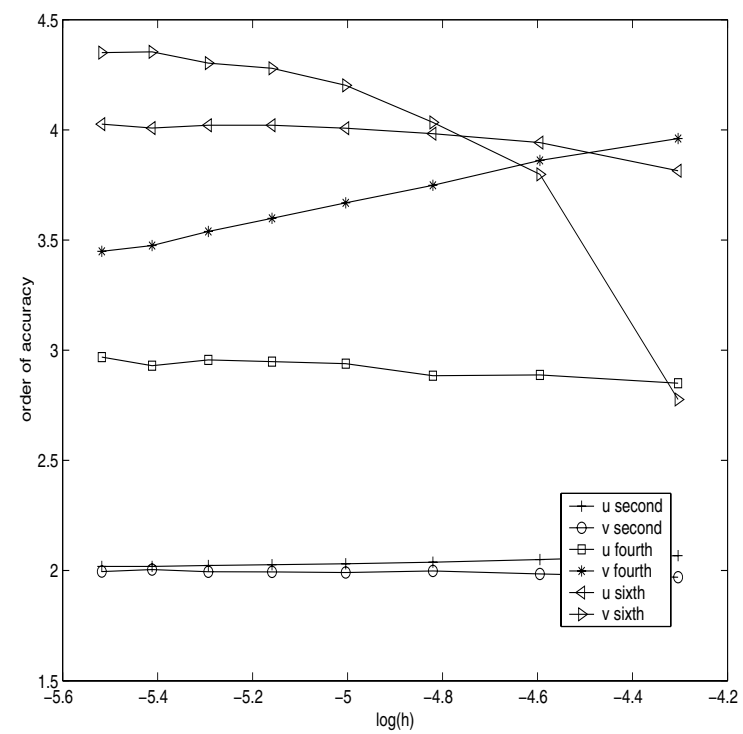

Fig. 16. Error at $t=0.2, a=1+0.8 \sin (\pi x), b=-1+0.8 \sin (\pi x), f=\sin (2 \pi x), g=-f$.

In Figs. 17 and 18, an initial perturbation in $v$ decreases and finally vanishes as $t$ increases. The disturbance is removed by the dissipation terms. In the continuous case, the perturbation propagates along with the rest of the solution.

By choosing $f=\sin (1.5 \pi x)$ and $g=-f$ we get a discontinuity that travels through the right boundary into the solution of $v$. In Figs. 19 and 20 we see that the conservative method with artificial dissipation reduces the perturbations caused by the discontinuity more than the skew-symmetric method.

\section{CONCLUSIONS}

We have determined a new type of artificial dissipation that depends on the variable coefficients and it's derivatives and the size of the grid. Our basic assumption is that the variable coefficients and the continuous solution are smooth. The artificial dissipation is constructed by expressing the conservative formulation as a skew-symmetric formulation by adding an artificial dissipation term to it. We have shown that it is possible to make the conservative method strictly stable by adding an artificial dissipation term without destroying the accuracy. We have also shown that strict stability cannot be obtained by using the skew-symmetric formulation only. 


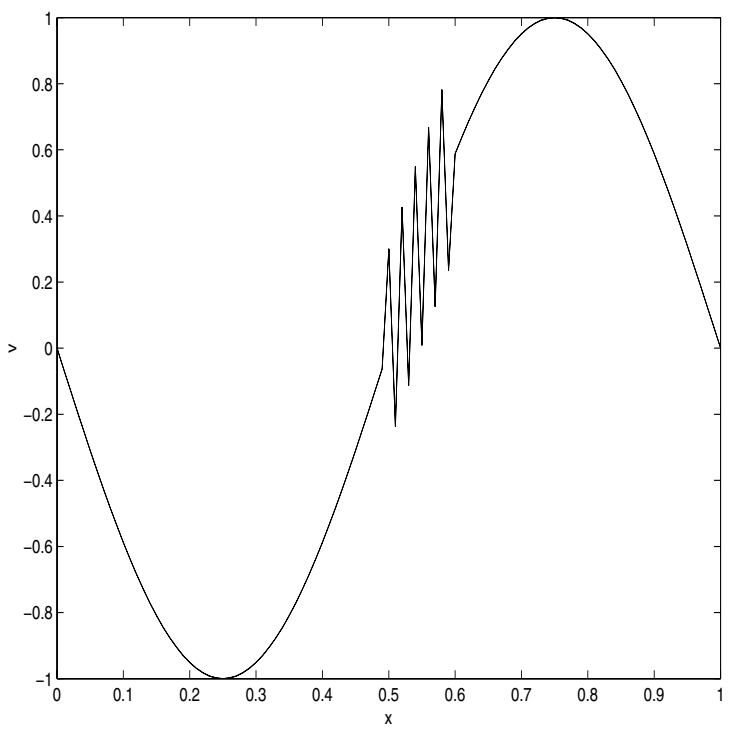

Fig. 17. $v$ at $t=0$. Sixth order case, $a=1+0.8 \sin (\pi x), b=-1+0.8 \sin (\pi x), f=$ $\sin (2 \pi x), g=-f+$ perturbation, $N=101$.

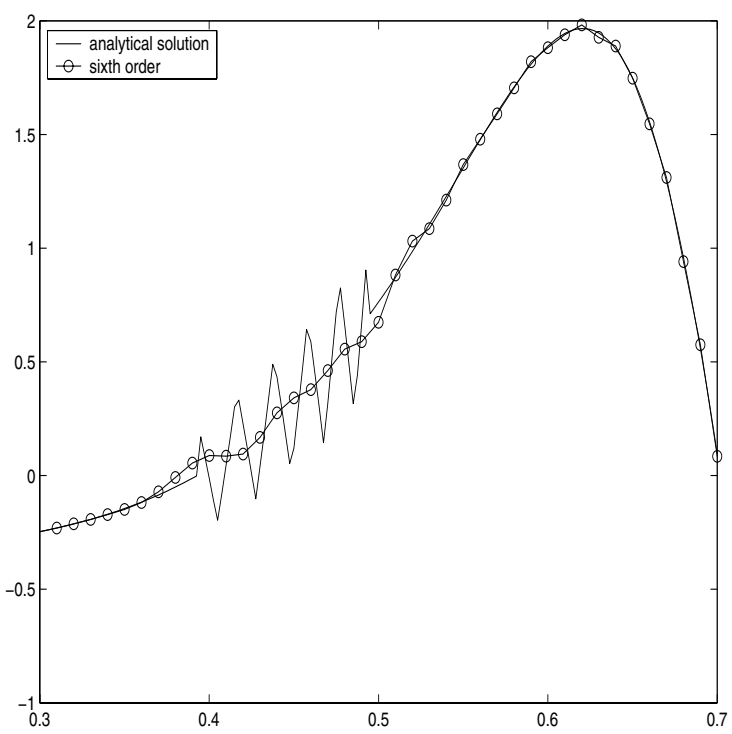

Fig. 18. $v$ at $t=0.5$. Sixth order case, $a=1+0.8 \sin (\pi x), b=-1+0.8 \sin (\pi x), f=$ $\sin (2 \pi x), g=-f+$ perturbation, $N=101$. 


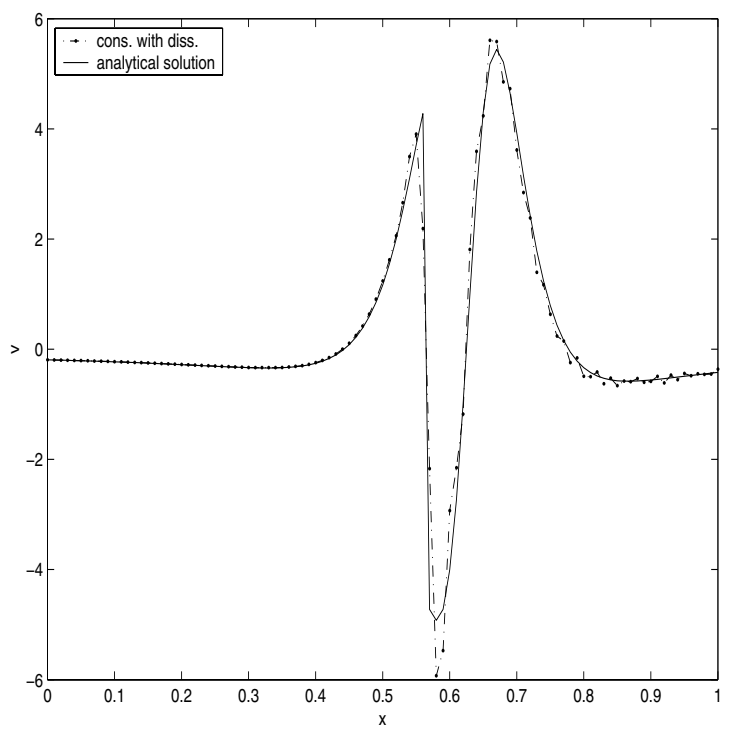

Fig. 19. $v$ at $t=1.0$. Conservative method with dissipation term, sixth order case, $a=1+$ $0.8 \sin (\pi x), b=-1+0.8 \sin (\pi x), f=\sin (1.5 \pi x), g=-f, N=100$.

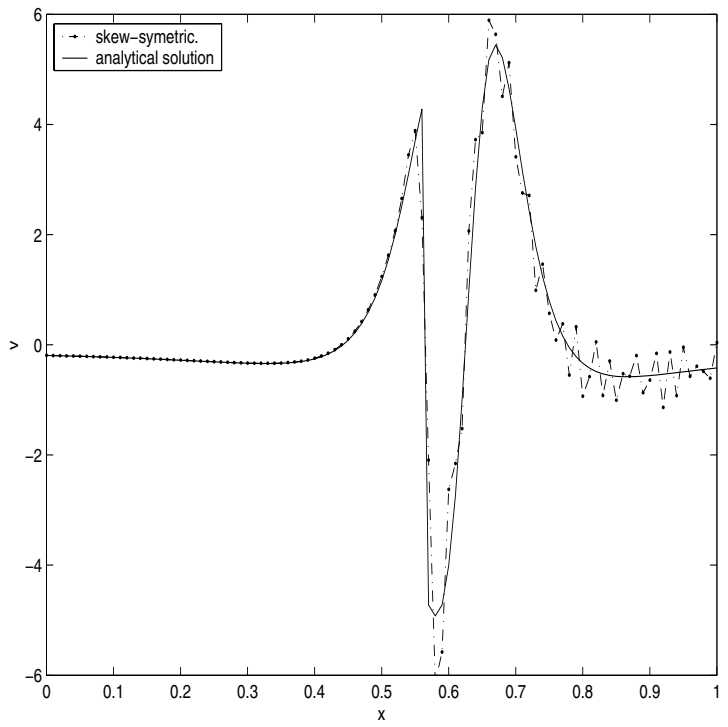

Fig. 20. $v$ at $t=1.0$. Skew-symmetric method with dissipation term, sixth order case, $a=$ $1+0.8 \sin (\pi x), b=-1+0.8 \sin (\pi x), f=\sin (1.5 \pi x), g=-f, N=100$. 


\section{APPENDIX A. SBP-OPERATORS}

A SBP-operator with diagonal norm [4] is on the form

$$
D=\frac{1}{\Delta x}\left(\begin{array}{cccc}
A & & & \\
& d & & \\
& & \ddots & \\
& & d & \\
& & & B
\end{array}\right) .
$$

$A=A(n, m)$ is a matrix who takes care of derivatives close to the left boundary. $B=-\operatorname{rot}\left(A, 180^{\circ}\right)$ is of the same size as $A$ but deals with derivatives on the right boundary. The value of $n$ and $m$ depends on the order of accuracy of the SBP-operator. The derivatives in the inner is taken care of by the row vector $d$. $d$ :s wideness depends on what SBP-operator we use. A SBP-operator can be written as $D=P^{-1} Q$, where $P$ in our case is a diagonal matrix.

Second order accurate difference operator:

$$
\begin{gathered}
A=\left(\begin{array}{ll}
-1 & 1
\end{array}\right) \\
d=\frac{1}{2}\left(\begin{array}{lll}
-1 & 0 & 1
\end{array}\right) \\
P=\Delta x \operatorname{diag}\left(\begin{array}{lllll}
\frac{1}{2} & 1 & \ldots & 1 & \frac{1}{2}
\end{array}\right)
\end{gathered}
$$

Fourth order accurate difference operator:

$$
\begin{aligned}
& A=\left(\begin{array}{rrrrrr}
-\frac{24}{17} & \frac{59}{34} & -\frac{4}{17} & -\frac{3}{34} & 0 & 0 \\
-\frac{1}{2} & 0 & \frac{1}{2} & 0 & 0 & 0 \\
\frac{4}{43} & -\frac{59}{86} & 0 & \frac{59}{86} & -\frac{4}{43} & 0 \\
\frac{3}{98} & 0 & -\frac{59}{98} & 0 & \frac{32}{49} & -\frac{4}{49}
\end{array}\right) \\
& d=\frac{1}{12}\left(\begin{array}{lllll}
1 & -8 & 0 & 8 & -1
\end{array}\right) \\
& P=\Delta x \operatorname{diag}\left(\begin{array}{llllllll}
\frac{17}{48} & \frac{59}{48} & 1 & \ldots & 1 & \frac{43}{48} & \frac{49}{48}
\end{array}\right)
\end{aligned}
$$


Sixth order accurate difference operator:

$$
\begin{aligned}
& A_{1: 6,1: 3}=\left(\begin{array}{ccc}
-\frac{21600}{13649} & \frac{43200}{13649} c-\frac{7624}{40947} & -\frac{172800}{13649} c+\frac{715489}{81894} \\
-\frac{8640}{12013} c+\frac{7624}{180195} & 0 & \frac{86400}{12013} c-\frac{57139}{12013} \\
\frac{17280}{2711} c-\frac{715489}{162660} & -\frac{43200}{2711} c+\frac{57139}{5422} & 0 \\
-\frac{25920}{5359} c+\frac{187917}{53590} & \frac{86400}{5359} c-\frac{745733}{64308} & -\frac{86400}{5359} c+\frac{176839}{16077} \\
\frac{34560}{7877} c-\frac{147127}{47262} & -\frac{129600}{7877} c+\frac{91715}{7877} & \frac{172800}{7877} c-\frac{242111}{15754} \\
-\frac{43200}{43801} c+\frac{89387}{131403} & \frac{172800}{43801} c-\frac{240569}{87602} & -\frac{259200}{43801} c+\frac{182261}{43801}
\end{array}\right) \\
& A_{1: 6,4: 6}=\left(\begin{array}{ccc}
\frac{259200}{13649} c-\frac{187917}{13649} & -\frac{172800}{13649} c+\frac{735635}{81894} & \frac{43200}{13649} c-\frac{89387}{40947} \\
-\frac{172800}{12013} c+\frac{745733}{72078} & \frac{129600}{12013} c-\frac{91715}{12013} & -\frac{34560}{12013} c+\frac{240569}{120130} \\
\frac{86400}{2711} c-\frac{176839}{8133} & -\frac{86400}{2711} c+\frac{242111}{10844} & \frac{25920}{2711} c-\frac{182261}{27110} \\
0 & \frac{43200}{5359} c-\frac{165041}{32154} & -\frac{17280}{5359} c+\frac{710473}{321540} \\
-\frac{86400}{7877} c+\frac{165041}{23631} & 0 & \frac{8640}{7877} c \\
\frac{172800}{43801} c-\frac{710473}{262806} & -\frac{43200}{43801} c & 0
\end{array}\right)
\end{aligned}
$$

$$
A_{1: 6,7: 9}=\left(\begin{array}{ccc}
0 & 0 & 0 \\
0 & 0 & 0 \\
0 & 0 & 0 \\
\frac{72}{5359} & 0 & 0 \\
-\frac{1296}{7877} & \frac{144}{7877} & 0 \\
\frac{32400}{43801} & -\frac{6480}{43801} & \frac{720}{43801}
\end{array}\right)
$$

where $c=\frac{342523}{518400}$.

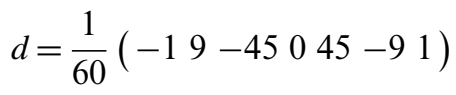




$$
\begin{aligned}
P=\Delta x & \operatorname{diag}\left(\begin{array}{cccccccc}
\frac{13649}{43200} & \frac{12013}{8640} & \frac{2711}{4320} & \frac{5359}{4320} & \frac{7877}{8640} & \frac{43801}{43200} & 1 & \ldots \\
1 & \frac{43801}{43200} & \frac{7877}{8640} & \frac{5359}{4320} & \frac{2711}{4320} & \frac{12013}{8640} & \frac{13649}{43200}
\end{array}\right)
\end{aligned}
$$

\section{APPENDIX B. ANALYTICAL SOLUTION}

Consider following initial value problem

$$
u_{t}+(a u)_{x}=0, \quad u(x, 0)=f(x)
$$

where $a=a(x)$. We can solve (45) by transforming this partial differential equation to three ordinary differential equations. This is done by adding two new variables, $y$ and $s$, where we define $y$ as $\frac{d u}{d y}=-a_{x} u$ and $s$ as $x=s$ when $t=0$. The three new equations are

$$
\frac{d t}{d y}=1, \quad \frac{d x}{d y}=a, \quad \frac{d u}{d y}=-a_{x} u
$$

This gives the solution $u=f(s) e^{\int_{y_{0}}^{y}-a_{x} d y}$ where $a=a(x), x=x(y, s) . s$ can be determined from

$$
\int_{0}^{x} \frac{1}{a} d x=y+\int_{0}^{s} \frac{1}{a} d x
$$

Using the relation $y=t$ from the first equation in (46) the final expression becomes

$$
u=f(s) e^{\int_{0}^{t}-a_{x} d y} .
$$

$s$ can be seen as a characteristic line to a point in the analytical solution. When we express $s$ explicity from (47) we do not always get $s=x$ when $t=$ 0 , for instance for some periodic $a(x)$. For that reason this solution only holds for some $a(x)$.

In this paper we wish to solve compute the solution to (18) and (19). Since $a>0$ and $b<0$, points in solution of $u$ move towards the right boundary. And consequently points in the solution of $v$ move in opposite direction. To calculate $u$ in a certain point at a certain time, we determine where the point were located at $t=0$, follow that specific characteristic line back in time to the boundary, and note the time. With known $x$ and $t$ at the boundary we can track the point through the solution of $v$ to the 
other boundary. This procedure is repeated until $t=0$. The value of $u$ in this specific point, for the case in Fig. B is then

$$
\begin{aligned}
u\left(x_{n}, t_{n}\right) & =\alpha v\left(0, t_{a}\right) e^{\int_{t_{a}}^{t_{n}}-a_{x} d y} \\
v\left(0, t_{a}\right) & =\beta u\left(1, t_{b}\right) e^{\int_{t_{b}}^{t_{a}}-a_{x} d y} \\
u\left(1, t_{b}\right) & =\alpha v\left(0, t_{c}\right) e^{\int_{t_{c}}^{t_{b}}-a_{x} d y} \\
v\left(0, t_{c}\right) & =g\left(s_{v}\left(0, t_{c}\right)\right) e^{\int_{0}^{t_{c}}-a_{x} d y} .
\end{aligned}
$$

By combining the formulas above we obtain the final expression,

$$
u\left(x_{n}, t_{n}\right)=\alpha^{2} \beta g\left(s_{v}\left(0, t_{c}\right)\right) e^{\int_{0}^{t_{n}}-a_{x} d y} .
$$

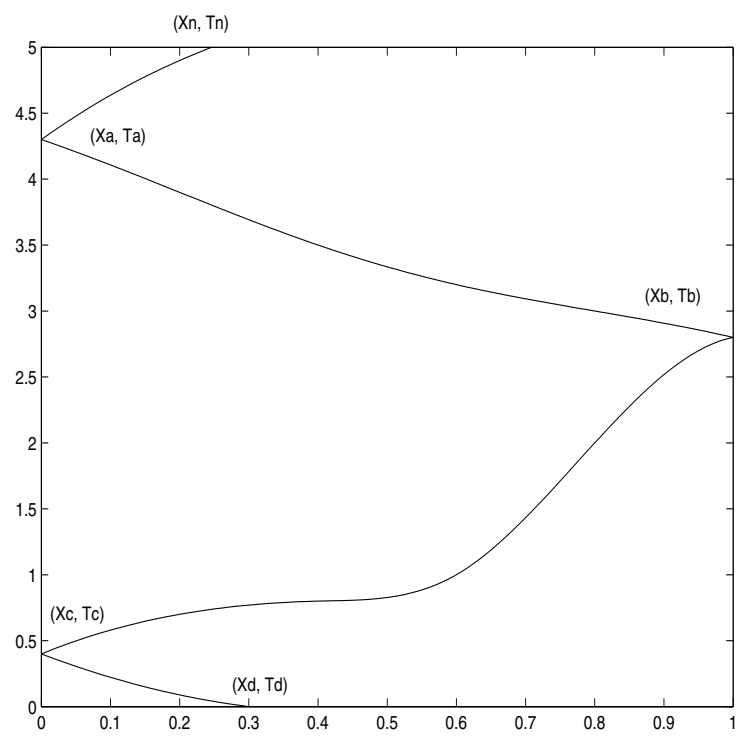

Fig. B. Characteristic lines for a point $p=\left(x_{n}, t_{n}\right)$ in $u$.

\section{REFERENCES}

1. Bretz, B., Forsberg, K., and Nordström, J. (2000). High Order Finite Difference Approximations of Hyperbolic Problems, FFA TN 2000-09, Stockholm.

2. Carpenter, M. H., and Gottlieb, D. (1996). Spectral methods on arbitrary grids. J. Comput. Phys. 129, 74-86. 
3. Carpenter, M. H., Gottlieb, D., and Abarbanel, S. (1994). The stability of numerical boundary treatments for compact high-order finite difference schemes. J. Comput. Phys. 108(2), 272-295.

4. Carpenter M. H, Nordström, J., and Gottlieb, D. (1999). A stable and conservative interface treatment of arbitrary spatial accuracy. J. Comput. Phys. 148, 341-365.

5. Gottlieb, D., and Hesthaven, J. S. (2001). spectral methods for hyperbolic problems. J. Comput. and Appl. Math. 128, 83-131.

6. Gustavsson, B., Kreiss, H.-O, and Oliger, J. (1995). Time Dependent Problems and Difference Methods. John Wiley\&Sons, New York.

7. LeVeque, R. J. (1990). Numerical Methods for Conservation Laws, Birkhäuser Verlag, Basel, Boston, Berlin.

8. Mattson K., and Nordström, J. (2004). Finite difference approximations of second derivatives on summation by parts form. J. Comput. Phys., 199, 503-540.

9. Mattson, K., Svärd, M. and Nordström, J. (2004). Stable and accurate artificial dissipation. J. Sci. Comput. 21(1), 57-79.

10. Strand, B. (1994). Summation by parts for finite difference approximations for $d / d \mathrm{x}$. J. Comput. Phys. 110, 47-67. 